K. Harada

Nagoya Math. J.

Vol. 33 (1968), 117-127

\title{
A CHARACTERIZATION OF THE ZASSENHAUS GROUPS
}

\author{
KOICHIRO HARADA
}

\section{Introduction}

A doubly transitive permutation group $\mathbb{B}$ on the set of symbols $\Omega$ is called a Zassenhaus group if is satisfies the following condition: the identity is the only element leaving three distinct symbols fixed.

The Zassenhaus groups were classified by H. Zassenhaus [14], W. Feit [3], N. Ito [7], and M. Suzuki [9]. There have been several characterizations of the Zassenhaus groups. Namely M. Suzuki [10] has proved that if a non abelian simple group \&f has a non-trivial partition then $\mathscr{S}$ is isomorphic with one of the groups $\operatorname{PSL}(2, q)$ or $\mathbf{S z}\left(2^{n}\right)$. Since each of the groups PSL $(2, q), \mathbf{S z}\left(2^{n}\right)$ has a non-trivial partition, a theorem of Suzuki characterizes them.

In this paper we shall characterize the Zassenhaus groups as permutation groups by a property of the centralizer of their involutions.

Let $\mathscr{S}$ be a finite permutation group on a set of $n$ symbols $\Omega=\{1,2$, ...n\}. For every $i(0 \leq i \leq n)$, we define a subset $\mathfrak{F}_{i}$ of $\mathbb{B}$ in the following way:

$$
\mathfrak{E}_{i}=\{G \in \mathbb{S} \mid G \text { leaves exactly } i \text { distinct symbols fixed }\} .
$$

Clearly each $\mathfrak{C}_{i}$ is a union of some conjugate classes of $\mathfrak{S}$. In particular $\mathfrak{E}_{n}=\{1\}$. A subset $\mathfrak{C}_{i}$ may be empty for some $i$. We shall set a following condition:

$\left(c_{i}\right)$ there exists an involution $I^{(i)} \in \mathfrak{E}_{i}$ such that the centralizer $\mathfrak{夭}_{\mathbb{B}}\left(I^{(2)}\right)$ of $I^{(i)}$ in $\mathbb{S}$ is contained in $\mathfrak{F}_{i} \cup\{1\}$.

It is easy to see that every conjugate element $J$ of $I^{(i)}$ has the same property as $I^{(i)}$. As a matter of fact, the linear fractional groups $\operatorname{PSL}(2, q)$ and Suzuki's simple groups $\mathbf{S z}\left(2^{m}\right)$ satisfy one of the conditions $\left(c_{0}\right),\left(c_{1}\right)$ or

Received Nov. 14, 1967. 
$\left(c_{2}\right)$. More strongly the above mentioned simple groups satisfy the following condition $\left(a_{i}\right)$ for $i=0,1$ and 2 :

$\left(a_{i}\right)$ for every element $A$ of $\mathfrak{E}_{i}$, the centralizer $\mathfrak{E}_{\mathscr{G}}(A)$ is contained in $\mathfrak{C}_{i} \cup\{1\}$.

Other than PSL $(2, q)$ and $\mathbf{S z}\left(2^{m}\right)$, the Mathieu group $\mathfrak{M}_{22}$ of degree 22 satisfies the condition $\left(a_{1}\right)$. If we consider the Mathieu group $\mathfrak{M}_{11}$ as a permutation group of degree 12 , then $\mathfrak{M}_{11}$ satisfies $\left(a_{2}\right)$. It is interesting to investigate the structure of $\& s$ satisfying the condition $\left(a_{i}\right)$ for some $i$. It seems, however, difficult to treat.

Now we state our result.

Theorem. Let \&s be a doubly transitive permutation group on $\Omega$. Let us assume that \&s satisfies the condition $\left(c_{i}\right)$ for some $i$. Then \&s is isomorphic with one of the groups PSL $(2, q)$ or $\boldsymbol{S} \boldsymbol{z}\left(2^{m}\right)$, or \&s has a regular normal subgroup.

Remark. There exists a non solvable exactly doubly transitive group satisfying $\left(c_{1}\right)$ (see Zassenhaus [15]). Therefore the last statement of the theorem is necessary even if we assume that $G$ is non solvable.

The proof of the above theorem is divided into two cases;

case (1): $i=0$ or 1 ,

case (2): $i \geq 2$.

In case (1) our aim is to prove that the stabilizer $\mathfrak{H}$ of a symbol 1 has a normal subgroup $\Omega$ which is regular on $\Omega-\{1\}$. After it is proved, the elementary argument shows that $\& 5$ is a Zassenhaus group. In case (2) we shall apply an interesting work of N. Iwahori [8] who has investigated the structure of groups of positive type. In later section we shall recall his definitions and results. Using a result of $\mathrm{N}$. Iwahori we shall prove that a Sylow 2-subgroup $\mathfrak{S}$ of $\mathbb{S}$ is a dihedral group and the centralizer $\mathfrak{S}_{\mathscr{S}}(I)$ of a central involution $I$ of $\subseteq$ has an abelian normal 2-complement. By a theorem of D. Gorenstein-J. Walter [6], we can easily prove our theorem.

Our notation is mostly standard. Denote by $(\mathscr{S}, \Omega)$ a permutation group on a set $\Omega$ of $n$ symbols $\{1,2, \cdots, n\}$. If a subgroup $\mathfrak{A}$ of $\mathscr{S}$ acts on a subset $\Delta$ of $\Omega$, we denote a permutation group induced by $\mathfrak{A}$ on $\Delta$ by $\left(\mathfrak{A}^{\Delta}, \Delta\right)$ or simply by $\mathfrak{A}^{\Delta}$. $\mathfrak{U}^{\Delta}$ is a homomorphic image of $\mathfrak{A}$. The normalizer or the centralizer of a subset $\mathfrak{X}$ of $\mathfrak{S}$ is denoted by $\mathfrak{A}_{\mathfrak{S}}(\mathfrak{X})$ or $\mathfrak{S}_{\mathscr{S}}(\mathfrak{X})$ respect- 
ively, or simply by $\mathfrak{A}(\mathfrak{X}), \mathfrak{C}(\mathfrak{X})$ if no confusion seems to occur. The image of a symbol $j$ by the action of an element $G$ of $\mathbb{S}$ is denoted by $j^{G}$. $|\mathfrak{M}|$ is the cardinality of a certain set $\mathfrak{M}$. All groups considered are finite.

\section{Proof of Theorem}

\section{Preliminary Lemmas}

First we shall prove two lemmas.

Lemma 1. Let \&s be a permutation group satisfying the condition $\left(c_{i}\right)$ for some $i$. If all the involutions of \&s are contained in a single conjugate class, then involutions are only elements which have transpositions in their cycle decompositions.

Proof. Let $A$ be an element of $\& 5$ whose cycle decomposition contains a transposition:

$$
A=(a, b) \cdots \cdots
$$

Then $A$ is a 2-singular element. Therefore $A$ is commutative with a certain involution $I$ which is conjugate to $I^{(i)}$ by assumption. If $A^{2}$ is not the identity element of $\&$, then $A^{2}$ is commutative with $I$ and $A^{2}$ leaves at least $i+2$ symbols invariant. This is impossible. This follows the lemma.

LEMMA 2. Let \&s be a doubly transitive permutation group satisfying the condition $\left(c_{i}\right)$ for some $i$. If all the involutions of $\&$ are contained in a single conjugate class, then the order of the centralizer $\mathfrak{C}_{ङ}(I)$ of any involution $I$ is equal to $n-i$.

Proof. Let $\beta(G)$ denote the number of transpositions in the cycle decomposition of an element $G$ of $\circlearrowleft 5$. Then by a theorem of $G$. Frobenius [5] we get a following equality:

$$
\sum_{G \in \mathscr{B}} \beta(G)=|\&| / 2 \text {. }
$$

By Lemma $1, \beta(G)>0$ if and only if $G$ is an involution of \&s. Hence

$$
\beta(I) \cdot|\circlearrowleft| S|/| \mathfrak{S}(I)|=| \mathfrak{S} \mid / 2 \text {. }
$$

On the other hand, since an involution $I$ has $n-i / 2$ transpositions we get easily

$$
|\mathfrak{S}(I)|=n-i
$$


2. Case $(1): i=0$ or $i=1$.

Let $\&$ be a non-solvable doubly transitive group on $\Omega$ satisfying the condition $\left(c_{i}\right)$ for $i=0$ or $i=1$. Assume that \&s has no regular normal subgroup. Denote by $\mathfrak{S}$ the stabilizer of the symbol 1 and by $\Re$ the stabilizer of the symbols 1 and 2 . Let $J$ be an involution of $\mathscr{S}$ which is conjugate to $I^{(i)}$ where $i=0$ or 1 . By the double transitivity of \&s we can choose $J$ such that a cyclic decomposition of $J$ is (12) $\cdots, J$ is contained in the normalizer $\mathfrak{R}_{\mathscr{S}}(\mathfrak{\Re})$ of $\mathfrak{\Re}$ in $\mathfrak{S}$. Therefore $J$ induces an automorphism of order 2 on $\Re$. By the condition $\left(c_{0}\right)$ or $\left(c_{1}\right), J$ has no fixed element in $\Re$. Hence $\Re$ is an abelian group of odd order. $J$ inverts every element of $\Re$.

Lemma 3. If $i=0$ or 1 , then all the involutions of (5) are contained in a single conjugate class.

Proof. Let $J_{1}$ and $J_{2}$ be two involutions of \&s. By the double transitivity of $\&$, there exists an element $A$ such that

$$
\begin{aligned}
& J_{1}=(a b) \cdots \cdots \\
& J_{2}^{A}=(a b) \cdots \cdots
\end{aligned}
$$

Hence the element $B=J_{1} J_{2}^{A}$ is contained in a suitable conjugate subgroup $\Re^{G}$ of $\mathfrak{R}$. Therefore the order of $B$ is odd. This implies $J_{1}$ and $J_{2}^{A}$ are conjugate to each other in $\Re^{G}$. Thus we have proved our lemma.

If $i=1$, then by Lemma 3 every involution has the same property as $I^{(1)}$. Therefore we can choose an involution $I$ which is conjugate to $I^{(1)}$ and leaves the symbol 1 fixed.

Lemma 4. If $i=1$, then $\mathfrak{S}=\mathfrak{S}_{\mathfrak{B}}(I) \Re$. Furthermore, every involution of $\mathfrak{H}$ is written in a form $I^{K}$ where $K$ is an element of $\Re$.

Proof. Let $I_{1}$ and $I_{2}$ be two involutions of $\mathfrak{H}$. Then by Lemma 3 $I_{1}^{G}=I_{2}, G \in \mathbb{S}$. Therefore $1^{G^{-1} I_{1} G}=1^{I_{2}}=1$. Hence $I_{1}$ leaves the symbol $1^{G^{-1}}$ fixed. Since $I_{1} \in \mathfrak{F}_{1}, 1^{G^{-1}}=1$. Hence $G \in \mathfrak{S}$. In particular $\mathfrak{S}_{\mathfrak{G}}(I) \subset \mathfrak{S}$. By Lemma 2 and Lemma 3, we have $|\Subset(I)|=n-1$. Since the order of $\mathfrak{H}$ is $(n-1) \cdot|\mathfrak{R}|$ and $\mathfrak{S}(I) \cap \Re=\{1\}$ by the condition $\left(c_{1}\right)$, we conclude $\mathfrak{S}=\mathfrak{C}(I) \cdot \Re$. Thus we have proved our lemma.

Lemma 5. If $i=0$ or 1 , then $\left[\mathfrak{R}_{\mathfrak{G}}(\Re): \Re\right]=2$. 
Proof. Let $\Delta$ be a set of symbols of $\Omega$ which are left fixed individually by every element of $\Re$. By a theorem of Witt [13], $\mathfrak{N}(\Re) / \Re$ is considered as a doubly transitive permutation group on $\Delta$. We can easily prove that this permutation group is exactly doubly transitive. Therefore we can conclude that $|\Delta|=q^{s}$ where $q$ is a prime number. Assume $q=2$. Then a Sylow 2-subgroup of $\mathfrak{R}(\Re)$ is an elementary abelian 2-group of order $2^{s}$. Since every involution of $\mathfrak{R}(\Re)$ inverts every element of $\mathfrak{R}$, we conclude $s=1$. This implies $[\mathfrak{N}(\Re): \Re]=2$. Next assume that $q$ is odd. Since $|\mathfrak{H} \cap \mathfrak{R}(\mathfrak{R}) / \mathfrak{R}|=q^{s}-1$. There exists an involution $I_{1}$ of $\mathfrak{H}$ which acts on $\mathfrak{K}$. Clearly $i=1$ and $n$ =odd in this case. Since $\Re$ is an abelian group, all the involutions of $\mathfrak{S}$ act on $\mathfrak{A}$ by Lemma 4. Therefore if a Sylow 2subgroup of $\mathfrak{S}$ has at least two involutions, then there exists an involution $I_{2}$ which acts trivially on $\Re$, which is impossible by the condition $\left(c_{1}\right)$. Thus a Sylow 2-subgroup of $\mathfrak{S}$ has only one involution. Since $n$ is odd, a Sylow 2-subgroup of $\mathfrak{S S}$ is isomorphic to that of $\mathfrak{H}$ and has only one involution. Hence a Sylow 2-subgroup of $\mathscr{S}$ is either cyclic or generalized quaternion group. Therefore $\&$ S has a regular normal subgroup (Burnside [2], Brauer-Suzuki [1], Feit-Thompson [4]). This is impossible. Thus we have proved our lemma.

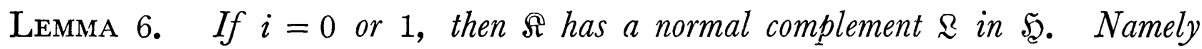
$\mathfrak{K}=\mathfrak{L} \cdot \mathfrak{R}, \mathfrak{Q} \cap \mathfrak{\Re}=1$.

Proof. By Burnside's splitting theorem, it suffices to show that $\mathfrak{N}_{\mathfrak{F}}\left(\Re_{p}\right)=$ $\mathfrak{C}_{\mathfrak{g}}\left(\Re_{p}\right)=\Re$ for every Sylow $p$-subgroup $\Re_{p}$ of $\Re_{\text {. For, if }}$ so, $\Re_{p}$ is a Sylow $p$-subgroup of $\mathfrak{S}$ and it has a normal complement $\Omega_{p}$ in $\mathfrak{S}$. Put $\bigcap_{p|| \Omega \mid} \Omega_{p}=\mathfrak{\Omega}$. Clearly $\mathfrak{R}$ is a normal complement of $\mathscr{R}$ in $\mathfrak{g}$. Let $\Delta$ be a set of symbols of $\Omega$ which are left fixed individually by every element of $\Re_{p}$. Let us assume that $|\Delta| \geq 3$. By a theorem of Witt $\mathfrak{A}_{\mathscr{B}}\left(\mathfrak{\Re}_{p}\right)^{\Delta}$ is a doubly transitive group on 4 . Since $\widetilde{S}_{\mathscr{S}}\left(\mathfrak{R}_{p}\right)$ contains $\mathfrak{R}$ and $\mathfrak{R}$ leaves just two symbols 1,2 invariant by Lemma $5, \mathfrak{C}\left(\Re_{p}\right)^{\Delta}$ is a non-trivial normal subgroup of $\mathfrak{R}\left(\Re_{p}\right)^{\Delta}$ of odd order. By the double transitivity of $\mathfrak{N}\left(\Re_{p}\right)^{4}, \mathfrak{C}\left(\Re_{p}\right)^{\Delta}$ is transitive. Hence $|\Delta|$ is odd. Since $\Delta^{J}=\Delta$, an involution $J$ keeps at least one symbol unchanged. Hence $n=$ odd and $i=1$. The order of the group $\mathfrak{S} \cap \mathfrak{R}\left(\Re_{p}\right)$ is divisible by $|\Delta|-1$. Therefore $\mathfrak{S}$ has an involution which acts on $\mathfrak{R}_{p}$. Hence all the involutions of $\mathfrak{S}$ act on $\mathfrak{K}_{p}$ by Lemma 4. This implies that a Sylow 2-subgroup of $\mathbb{S}$ has only one involution. Hence $\mathbb{E}$ h has a regular 
normal subgroup. This is not the case. Hence $|\Delta|=2$. Hence $\mathfrak{R}\left(\Re_{p}\right)=$ $\langle J, \Re\rangle$. Therefore $\mathfrak{N}_{\mathfrak{W}}\left(\Re_{p}\right)=\mathfrak{S}_{\mathfrak{f}}\left(\Re_{p}\right)=\mathfrak{R}$. This yields our lemma.

Proposition 1. Let (S) be a doubly transitive permutation group satisfying the condition $\left(c_{i}\right)$ for $i=0$ or 1 . Then $\&$ is isomorphic with one of the groups $\operatorname{PSL}(2, q)$ or $S z\left(2^{m}\right)$, or is has a regular normal subgroup.

Proof. Assume that $\$ s$ has no regular normal subgroup. By Lemma 6 , $\mathfrak{T}$ has a normal subgroup $\mathfrak{Q}$ of order $n-1$ which is regular on $\Omega-\{1\}$. Therefore (s) admits a decomposition:

$$
\mathfrak{S}=\mathfrak{H}+\mathfrak{H} J \mathfrak{\Omega} \text {. }
$$

Every element of $\mathfrak{S}-\mathfrak{S}$ is uniquely expressed in a form $L^{\prime} K J L$ where $L^{\prime}, L \in \mathfrak{\Omega}, K \in \Re$. Next we shall show that $\mathfrak{R}$ is a T.I. set in (S). Since $\Re$ is an abelian subgroup, it suffices to show that the centralizer of any non-identity element of $\Re$ is equal to $\Re$. Let an element $K_{1} \in \mathfrak{R}$ is com-

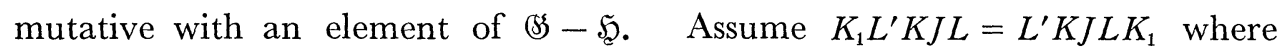
$K_{1}, K \in \Re, L^{\prime}, L \in \mathfrak{\Omega}$. Then $L^{\prime K_{1}^{-1}} K_{1} K J L=L^{\prime} K K_{1}^{-1} J L^{K_{1}}$. By the uniqueness of expression of an element of $\mathscr{S}-\mathfrak{H}$ we get $K_{1} K=K K_{1}^{-1}$. This implies $K_{1}=1$, since $\Re$ is an abelian group of odd order. If $K_{1}$ is commutative with an element $L$ of $\mathfrak{Q}$, then $K_{1}{ }^{J}$ is commutative with $L^{J} \in \mathbb{S}-\mathfrak{H}$. This is impossible by the above fact. Therefore $\mathfrak{R}$ is a T.I. set in $\mathfrak{S}$. Let us assume that an element $A \neq 1$ of $\Re$ keeps at least three distinct symbols, say $1,2,3$, unchanged. Then $A \in \Re \cap \Re^{H}$ where $1^{H}=1,2^{H}=3$. Therefore $\Re=\Re^{H}$ and $\Re$ keeps $1,2,3$ invariant. By Lemma 3 , this is impossible. Therefore $\mathbb{A S}$ is a Zassenhaus group. Since (S) has only one class of involutions and the order of any involution of \&s is $|\Omega|$ or $|\Omega|-1$, we get easily our proposition.

\section{Case (2): $i \geq 2$.}

First we shall recall a result of N. Iwahori [8].

Let $\mathfrak{S}$ be a permutation group on $\mathfrak{M}$. We call $\mathfrak{M}$ a $\mathfrak{S}$-space. Define a subset $\mathfrak{M}_{G}(G \in \mathbb{S})$ of $\mathfrak{M}$ as follows.

$$
\mathfrak{M}_{G}=\left\{m \in \mathfrak{M} \mid m^{G}=m\right\} .
$$

Definition 1. A permutation groups $\&$ on $\mathfrak{M}$ is of type $k$ if the following two conditions are satisfied;

(i) $\left|\mathfrak{M}_{G}\right|=k$, for every non identity element $G \in \mathscr{G}$, 
(ii) $\bigcap_{G \in \mathfrak{B}} \mathfrak{M}_{G}=\phi$, where $\phi$ denotes the empty set.

N. Iwahori's main result is the following theorem.

Theorem. If is admits a $\mathbb{S}$-space $\mathfrak{M}$ of type 2 , then (S) is isomorphic to one of the following groups:

(i) $A_{4}$ : the alternating group of degree 4 ,

(ii) $S_{4}$ : the symmetric group of degree 4 ,

(iii) $\mathfrak{A}_{5}$ : the alternating group of degree 5 or

(iv) a generalized dihedral group with dihedral Sylow 2-subgroups.

Here a generalized dihedral group is defined as follows. Let $\mathfrak{A}$ be an abelian group and $\tau$ be an automorphism of $\mathfrak{A}$ such that if $A \in \mathfrak{A}$, then $A^{\tau}=A^{-1}$, where $A^{\tau}$ denotes the image of $A$ by $\tau$. Under these conditions, holomorph of $\mathfrak{A}$ by $\tau$ is called a generalized dihedral group.

In order to prove his theorem, N. Iwahori has proved several lemmas. We shall quote one of them here.

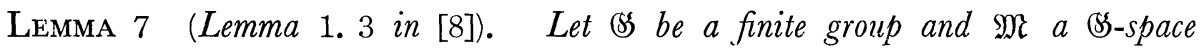
of type $k>0$. Let $A$ and $B$ be elements in $\mathbb{S}-\{1\}$ of orders $a$ and $b$ respectively. Assume that

(i) $A B=B A$, and

(ii) $a \neq b$ or $a=b \neq$ prime.

Then $\mathfrak{M}_{A}=\mathfrak{M}_{B}$.

Now we shall apply his argument to our case. Let \&s be a non solvable doubly transitive group on $\Omega$ satisfying the condition $\left(c_{i}\right)$ for $i \geq 2$. As in section 2, let us denote the stabilizer of the symbol 1 by $\mathfrak{H}$ and the stabilizer of two symbols 1 and 2 by $\Re$. $J$ is an involution of $\mathscr{S}$ which is conjugate to $I^{(\imath)}$. We can choose $J$ such that a cyclic decomposition of $J$ is $(12) \cdots$ In the rest of this paper we shall use the notation $I$ instead of $I^{(i)}$.

Lemma 8. The centralizer $\sqrt{5}(I)$ of $I$ admits a $\mathfrak{S}(I)$-space of positive type.

Proof. We may assume that $I$ leaves $i$ symbols, say $1,2, \cdots, i$ invariant. If $\mathfrak{S}(I)$ does not admit a $\mathfrak{S}(I)$-space of positive type, then by the condition $\left(c_{i}\right)$ every element $A \neq 1$ of $\mathfrak{C}(I)$ leaves just $i$ symbols $1,2, \cdots, i$ invariant. 
Clearly every conjugate subgroup of $\mathfrak{C}(I)$ does not also admit a $\mathfrak{C}(I)$-space of positive type. Therefore if $\mathfrak{C}\left(I^{G}\right) \cap \mathfrak{E}(I)>\{1\}$ then every element of $\mathbb{C}\left(I^{G}\right)$ leaves just $i$ symbols $1,2, \cdots, i$, invariant. Let $\Re_{2}$ be a Sylow 2-subgroup of $\mathscr{R}$ which is non-trivial by the condition $\left(c_{i}\right)(i \geq 2)$. Since $J$ acts on $\mathfrak{R}$, we may assume $\Re_{2}^{J}=\Re_{2}$. Put $\subseteq=\left\langle J, \Re_{2}\right\rangle$. Then there exists an involution $I_{1}$ of $\Re_{2}$ which is conjugate to $I$ and $\mathfrak{E}(J) \cap \mathfrak{S}\left(I_{1}\right) \supset \mathbb{Z}(\mathfrak{S})>\{1\}$. Thus every element of $\mathfrak{C}(J)$ leaves 1,2 , invariant. In particular $J$ leaves 1,2 invariant. This is impossible, since $J$ has a cyclic decomposition (12) - . Thus we have proved our lemma.

Lemma 9. $\mathbb{E}(I)$ is an elementary abelian 2-group or a generalized dihedral group.

Proof. Since $\mathfrak{C}(I)$ admits a $\mathfrak{C}(I)$-space of positive type, we may apply Lemma 7. Assume that $\mathfrak{E}(I)$ is not an elementary abelian 2-group. Let $\mathfrak{N}$ be a (normal) subgroup of $\mathfrak{C}(I)$ which is generated by all non-involutions of $\mathfrak{E}(I)$. By Lemma 7 , every element of $\mathfrak{R}$ leaves $1,2, \cdots i$ fixed. This implies that $\mathfrak{N}$ is a proper subgroup of $\mathfrak{C}(I)$. If $A$ is an element of $\mathfrak{C}(I)-\mathfrak{R}$, then $A^{2}=1$. Therefore $(A N)^{2}=1$ for $N \in \mathfrak{R}$. Hence $A^{-1} N A=N^{-1}$. Hence $\mathfrak{R}$ is an abelian subgroup of $\mathfrak{C}(I)$. If $B$ is another element of $\mathfrak{E}(I)-\mathfrak{R}$, then $B^{2}=1$ and the element $A B$ centralizes $\mathfrak{R}$. Hence $A \equiv B(\bmod \mathfrak{R})$. This implies that $[\mathfrak{C}(I): \mathfrak{N}]=2$. This follows our lemma.

Lemma 10. If $\mathfrak{C}(I)$ is not an elementary abelian 2-group, then $\mathfrak{C}(I)$ admits a $\mathfrak{C}(I)$-space of type 2.

Proof. Let $\Gamma$ be a subset of $\{1,2, \cdots, i\}$ consisting of elements left fixed by every element of $\mathfrak{C}(I)$. Put $\Delta=\{1,2, \cdots, i\}-\Gamma$. Since $\subseteq(I)$ admits a $\mathbb{C}(I)$-space of positive type, we have $|\Delta|=k \geq 1$. Let $r$ be the number of orbits of $\mathfrak{C}(I)$ on $\Omega-\Gamma=\mathfrak{M}$. Then

$$
r|\mathfrak{C}(I)|=|\mathfrak{M}|+k(|\mathfrak{C}(I)|-1)
$$

(Wielandt [13] p. 8 Ex. 3. 10).

Hence

$$
|\subseteq(I)|=\frac{|\mathfrak{M}|-k}{r-k}=\frac{n-(i-k)-k}{r-k}=\frac{n-i}{r-k} \leqq n-i .
$$

On the other hand, using a equality of Frobenius $\sum_{G \in \mathbb{S}} \beta(G)=|\&| / 2$ we get 


$$
\frac{n-i}{2} \cdot \frac{|\mathfrak{S}|}{|\mathfrak{S}(I)|} \leq|\mathfrak{S}| / 2
$$

Hence $|\mathfrak{S}(I)| \geq n-i$. Hence $|\mathfrak{S}(I)|=n-i, \quad r=k+1$ and $|\mathfrak{M}|=|\mathfrak{C}(I)|+k$. Since $\mathfrak{C}(I)$ has a normal subgroup $\Re$ of index 2 which leaves all the symbols of $\Delta$ fixed, $\Delta$ decomposes into $k / 2$ orbits of $\mathbb{C}(I)$. Since by the condition $\left(c_{i}\right)$ any element of $\mathfrak{R}$ has no fixed symbols on $\mathfrak{M}-\Delta$ each of the remaining orbits of $\mathfrak{C}(I)$ of $\mathfrak{M}-\Delta$ has length at least $\mathbb{C}(I) \mid / 2$ hence exactly $|\mathfrak{C}(I)| / 2$. Therefore we have the following equality.

$$
\frac{k}{2}+2=r=k+1
$$

Hence $k=2$. Thus we have proved our lemma.

Lemma 11. All the involutions of $\mathbb{s}$ are contained in a single conjugate class.

Proof. In the proof of Lemma 10, we have proved the equality $|\mathfrak{C}(I)|$ $=n-i$. This relation also holds when $\subseteq(I)$ is an elementary abelian 2group, because in proving the equality $|\mathfrak{C}(I)|=n-i$ we have used only the fact that $|\mathfrak{C}(I)|$ admits a $\mathscr{C}(I)$-space of positive type. Using a equality $\sum \beta(G)=\frac{1}{2}|\mathfrak{G}|$, we can easily prove that there exists no involution which is not conjugate to $I$.

Proposition 2. Let is be a doubly transitive permutation group satisfying the condition $\left(c_{i}\right)$ for $i \geq 2$. Then $i=2$ and $\mathbb{S S}$ is isomorphic to one of the groups $\operatorname{PSL}(2, q)$ where $q$ is a power of a certain odd prime, or \&s has a regular normal subgroup.

Proof. If $\mathrm{C}(I)$ is an elementary abelian 2-group, then by Lemma 11, (S5 is a (CIT)-group (Suzuki [11]). If (5s has a non trivial solvable normal subgroup, then \&f has a regular normal subgroup $\mathfrak{A}$. Assume that $\mathbb{E S}$ has no regular normal subgroup. By Theorem 5 of Suzuki [11] and the main theorem of Suzuki [9], of is isomorphic to one of the following groups: $\operatorname{LF}\left(2,2^{\alpha}\right)$, $S z\left(2^{\beta}\right)$, PSL $(2, q)$, PSL $(3,4)$ or $M_{9}$ (This is a group of order $9 \cdot 8 \cdot 7=720$, which is the projective group of one variable over the near-filed of 9 elements; Zassenhaus [14]). Since (5) is a (CIT) group, in the above mentioned groups only PSL $\left(2,2^{\alpha}\right)$ has elementary abelian 2-Sylow subgroups. If PSL $(2,4)$ $=\operatorname{PSL}(2,5)$ is considered as permutation group of degree 6 , PSL $(2,5)$ satisfies the condition $\left(c_{2}\right)$. If $2^{\alpha}>4$, the group $\operatorname{PSL}\left(2,2^{\alpha}\right)$ does not 
satisfy the condition $\left(c_{i}\right)$ for $i \geq 2$. Therefore $\mathscr{S} \cong \mathrm{PSL}(2,5)$. Next let us assume that $\mathfrak{C}(I)$ is not an elementary abelian 2-group. By Lemma 10 and by a theorem of $\mathrm{N}$. Iwahori, $\subseteq(I)$ is a generalized dihedral group with dihedral Sylow 2-subgroups. Since $I$ is a central involution of a certain Sylow 2-subgroup $\mathfrak{T}$ of $\mathbb{S} \mathfrak{S}$ by Lemma 11, $\mathfrak{T}$ is a dihedral group. Since $\mathfrak{C}(I)$ has a abelian normal 2-complement by a theorem of D. Gorenstein-J. Walter [6], \&S is isomorphic to one of the following groups: PSL $(2, q)$, PGL $(2, q)$ where $q$ is a power of an odd prime, or $\mathfrak{A}_{7}$ the alternating group of degree 7. Here we used the fact that \&s has not a solvable normal subgroup and that a group of odd order is solvable (W. Feit-J. Thompson [4]). On the other hand the group $\operatorname{PGL}(2, q)(q$ is odd) has two conjugate classes consisting of involutions. The group $\mathfrak{A}_{7}$ does not satisfy $\left(c_{i}\right)$, because $\mathfrak{A}_{7}$ has one class of involutions and a involution (12)(34) is commutative with (1324)(56). Hence (s) $\cong \operatorname{PSL}(2, q)$ ( $q$ is odd).

Combining Proposition 1 and Proposition 2 we have our main theorem stated in the introduction.

Remark. Recently M. Suzuki [12] has proved the following result.

Theorem. Let \&s be a finite group. Suppose that \&s contains a subgroup $\mathfrak{S}$ which satisfies the following two conditions:

(1) $\mathfrak{\mathfrak { T }}$ is a generalized dihedral group, and

(2) $\mathfrak{H}=\mathfrak{S}_{\mathscr{B}}(J)$ for any involution $J$ of the center of $\mathfrak{H}$.

Then, if $\&$ is not solvable, $\mathbb{S}$ contains a normal subgroup $\mathfrak{R}$ such that the order of $\mathfrak{R}$ is either odd or twise an odd number, and that $\mathbb{S} / \mathfrak{N} \cong \operatorname{PSL}(2, q)$ or $\operatorname{PGL}(2, q)$ for some prime power $q>3$.

If we use this theorem, our proof in case (ii) become rather short.

\section{REFERENCES}

[1] Brauer, R. and Suzuki, M., On finite groups of even order whose 2-Sylow group is a quaternion group, Proc. Nat. Acad. Sci., Vol. 45, (1959), pp. 1757-1759.

[2] Burnside, W., Theory of groups of finite order, Cambridge Univ. Press, (1911) (Second edition).

[ 3 ] Feit, W., On a class of doubly transitive permutation groups, Ill. J. Math., Vol. 4, (1960), pp. 170-186.

[4] Feit, W. and Thompson, J.G., Solvability of groups of odd order, Pac. J. of Math., Vol. 13, pp. 775-1028. 
[5] Frobenius, G., Über die Charactere der mehrfach transitiven Gruppen, S.B. Preuss. Akad. Wiss. 1904.

[6] Gorenstein, D. and Walter, J., On finite groups with dihedral Sylow 2-subgroups, Ill. J. Math., Vol. 6, (1962), pp. 553-593.

[ 7 ] Ito, N., On a class of doubly transitive permutation groups, Ill. J. Math., Vol. 6, (1962), pp. 341-352.

[ 8 ] Iwahori, N., On a property of a finite group, J. of Faculty of Sci., Univ. of Tokyo, Vol. 11, (1964), pp. 47-64.

[9] Suzuki, M., On a class of doubly transitive groups, Ann. of Math., Vol. 75, (1962), pp. 105-145.

[10] Suzuki, M., On a finite group with a partition, Arch. Math., Vol. 7, (1961), pp. 241-254.

[11] Suzuki, M., Finite groups with nilpotent centralizers, Trans. Amer. Math. Soc., Vol. 99, (1961), pp. 425-470.

[12] Suzuki, M., A characterization of the simple groups PSL(2, q), Jour. of Math. Soc. of Japan, Vol. 20, (1968), pp. 342 349.

[13] Wielandt, H., Finite permutation groups, Academic Press, New York, 1964.

[14] Zassenhaus, H., Kennzeichnung endlicher linearer Gruppen als Permutationsgruppen, Hamb. Abh. Vol. 11, (1936), pp. 17-40.

[15] Zassenhaus, H., Über endliche Fastkörper, Hamb. Abh., Vol. 11, (1936), pp. 187-220.

Nagoya University. 